Research Article

\title{
Study of Health-Related Quality of Life and Healthcare Utilization among Type 2 Diabetic Population in an Urban Area of Eastern Nepal
}

\author{
Sangita Shah $\left(\mathbb{D},{ }^{1}\right.$ Nilambar Jha, ${ }^{1}$ Deepak Kumar Yadav $\mathbb{D}^{1},{ }^{1}$ Prajjwal Pyakurel $\left(\mathbb{D},{ }^{1}\right.$ \\ Sanjib Kumar Sharma $\mathbb{D}^{2},{ }^{2}$ and Suman Bahadur Singh $\left(\mathbb{1}^{1}\right.$ \\ ${ }^{1}$ School of Public Health and Community Medicine, B.P.Koirala Institute of Health Sciences, Dharan, Nepal \\ ${ }^{2}$ Department of Internal Medicine, B.P.Koirala Institute of Health Sciences, Dharan, Nepal \\ Correspondence should be addressed to Sangita Shah; sangita.shah@bpkihs.edu
}

Received 8 September 2020; Revised 7 November 2020; Accepted 14 December 2020; Published 24 December 2020

Academic Editor: Alexander Schreiber

Copyright (c) 2020 Sangita Shah et al. This is an open access article distributed under the Creative Commons Attribution License, which permits unrestricted use, distribution, and reproduction in any medium, provided the original work is properly cited.

\begin{abstract}
Introduction. Diabetes mellitus is a major cause of morbidity and mortality and places huge burden on public health funding. Diabetes affects quality of life through associated complications, comorbidity, and disease burden. Consequently, people have frequent healthcare visits. This study assessed quality of life and healthcare utilization patterns among type 2 diabetic populations in an urban area of eastern Nepal. Methods. A cross-sectional study was conducted among 270 participants of age $\geq 20$ years with type 2 diabetes in Itahari using a semistructured questionnaire. A D-39 questionnaire was used to assess quality of life. Five wards were selected by systematic random sampling, and the population was proportionate according to the sample size. Multiple linear regressions were conducted to identify the factors associated with quality of life and its domains. Results. The highest mean score \pm SD was found in the domain anxiety and worry $(57.34 \pm 11.08)$. About $18.5 \%$ of the participants perceived extremely affected quality of life. Hypertension (55.55\%) was the most common comorbidity. Age, marital status, literacy, alcohol, disease duration, comorbidity, and complications were significantly associated with overall quality of life. In last 6 months of duration, 93.7\% had hospital visits. Among them, $8.1 \%$ had emergency visit and 5.9\% were admitted in the hospital. Conclusion. People with diabetes in this study were more affected in the domain anxiety and worry. The frequency of healthcare access and utilization in patients with type 2 diabetes was high. The quality of life among them could be improved by taking care on healthy behavior, comorbid conditions, and complications.
\end{abstract}

\section{Introduction}

Due to social and economic transition, leading to behavioral and metabolic risk factors, diabetes is leading as an emerging major public health problem in Nepal, with rising prevalence and its complications, especially in urban populations $[1,2]$. It is a chronic, incurable, costly, and largely preventable noncommunicable disease which is responsible for millions of deaths globally [3].

Diabetes has multidimensional effect in QOL such as social, physical, and role functioning, worries about the future, and emotional and general well-being [4]. For this, people living with diabetes require regular visits to several healthcare professionals and utilization of the service of clinics and outpatient centres [5, 6]. This study assist healthcare practitioners, institution, policy makers, and community people to better understand quality of life and healthcare utilization in type 2 diabetes mellitus [7].

This study is aimed to assess the health-related quality of life and healthcare utilization among type 2 diabetic population in an urban area of eastern Nepal.

\section{Materials and Methods}

A community-based cross-sectional study was conducted among 270 type 2 diabetes patients residing in Itahari, a 
submetropolitan city to assess their health-related quality of life and healthcare utilization pattern. The study population comprised of all the type 2 diabetes patients aged 20-80 years, diagnosed for more than 1 year of duration. Among the 26 wards of Itahari, 5 wards were selected by the systematic sampling method. The population in each ward was proportionate according to the sample size (270), and the final sample from each ward, i.e., 5, 8, 17, 16, and 23 was 133 , $62,29,24$, and 22, respectively. Patients were recruited through the nonprobability sampling method by taking help from the local health personnel such as Female Community Health Volunteer, Primary Healthcare Record, other private clinic/hospital, pharmacy, and other health agencies. After receiving informed consent, a face-to-face interview was conducted using pretested semistructured questionnaires and a standardized tool, the D-39 questionnaire. Permission was granted by the author to use this questionnaire. Privacy, confidentiality, and anonymity were assured and maintained.

All the collected data were entered in Microsoft Excel 2007, and the cleaned data were converted into Statistical Package for Social Sciences (SPSS) 11.5 version for statistical analysis. The descriptive data were expressed in terms of frequency, percentage, and mean with standard deviation along with graphical and tabular presentation of the data. The Student $t$-test and one-way ANOVA were applied to find out significant difference between health-related quality of life and other related variables at $95 \%$ confidence interval where $p<0.05$. The variables that were significant at $p<0.1$ from the bivariate analysis were considered for multivariate analysis using multiple linear regressions where the backward linear regression method was specified in order to find confounders and/or effect modifiers. The level of significance was $p<0.05$ for all the tests.

\section{Results and Discussion}

3.1. Results. Table 1 shows the demographic characteristics of type 2 diabetic patients in the study. The mean age \pm SD (range) of the respondents was 53.86 years $\pm 11.89(26-79)$. More than half $(54.4 \%)$ of the participants were obese followed by overweight (22.2\%).

Half of the participants $(52.6 \%)$ were suffering from diabetes for less than 5 years followed by $5-10$ years $(33.7 \%)$. Many of them (57.4\%) were having additional illness other than diabetes, and $18.9 \%$ were suffering from the complication of diabetes.

Out of the total participants, $55.5 \%$ had hypertension as the comorbidity (Figure 1).

Most of the participants were diagnosed (70.0\%) and treated $(82.20 \%)$ at a private health centre followed by BPKIHS (Figure 2).

Table 2 presents the domain-specific QOL scores among the study participants. The highest mean score (SD) was found in the domain anxiety and worry (57.34 \pm 11.08$)$.

Table 3 indicates that the factors such as age, ethnicity, marital status, alcohol, education, occupation, blood pressure, diabetes duration, comorbidity, complications, family history, treatment, history of ER visit, and hospital admission in last 6 months were significantly associated with overall QOL. Age was found to be an important factor for quality of life in the diabetes patients.

The results of multiple linear regressions revealed that age, marital status, education, alcohol status, duration of disease, comorbidity, complication, and emergency visit were significantly associated with overall QOL of patients with diabetes $(p<0.05)$. These variables accounted for $40.0 \%$ of the variance of the total HRQOL. Age, alcohol status, comorbidity, complication, and ER visit were found significant in the four domains, energy and mobility, diabetes control, anxiety and worry, and social overload. In every domain, participants with age $>50$ years increased the mean score by more than 3 (Table 4 ).

3.2. Discussion. Going through various literature studies, to the best of our knowledge, this is the first community-based cross-sectional study in an urban setting of Nepal that assessed domain-wise impact in quality of life among diabetes patients and explored the factors significantly affected their QOL.

More than half of the participants $(57.4 \%)$ in this study suffered from at least one comorbidity which agrees with the community-based cross-sectional study done in Khartoum, Sudan [8]. Hypertension was found to be the most common comorbidity (55.55\%) in this study. Among those having comorbidity, $96.8 \%$ had hypertension. One of the studies done in a tertiary care diabetes centre in Karachi-Pakistan found that $57.2 \%$ of the participants were hypertensive [9]. This finding was also in agreement with the study done in polyclinic of Benghazi City [10]. This could conclude that diabetes and hypertension are closely related morbidities.

Treatment from private health sector was sought by $82.2 \%$ of diabetic patients. One of the studies done in Mumbai, India, among urban slums found that $81 \%$ of participants seek treatment from private health sector [11]. This could be linked with dissatisfaction of public hospital services and easy access to the private health centre.

As regular blood sugar examination is an important part of the treatment in diabetes patients, about $94 \%$ of the participants in this study visited the hospital for it. This act as a mirror in the finding of the study done among slums in Mumbai, where $75 \%$ of the participants visited healthcare in last 6 months [11]. The increase in awareness about the disease is the reason for higher percentage of participants visiting health centres, but the difference between both studies reveals that people of Itahari, submetropolitan city, are economically more strong.

The highest mean score (SD) was found in the domain anxiety and worry $(57.34 \pm 11.08)$. This reveals that most of the participants' QOL is affected in this domain. A crosssectional study performed at two Basic Healthcare Units (BHU) in the western district of the City Health Department of Ribeirão Preto in 2012 [12] showed the highest mean score in social overload dimension and one of the hospital-based study in Kathmandu, Nepal [13]; quality of life was mostly affected in the domain physical health. These both findings are contradicted with the study finding. It can be predicted 
TABLE 1: Demographic characteristics of the participants $(n=270)$.

\begin{tabular}{|c|c|c|c|}
\hline Characteristics & Categories & Frequency & Percentage (\%) \\
\hline \multirow{3}{*}{ Age (years) } & $20-40$ & 37 & 13.7 \\
\hline & $41-60$ & 159 & 58.9 \\
\hline & $61-80$ & 74 & 27.4 \\
\hline \multicolumn{4}{|c|}{ Mean age \pm SD (range) 53.86 years \pm 11.89 (26-79) } \\
\hline \multirow{2}{*}{ Sex } & Male & 136 & 50.4 \\
\hline & Female & 134 & 49.6 \\
\hline \multirow{4}{*}{ Marital status } & Married & 229 & 84.8 \\
\hline & Widow & 31 & 11.5 \\
\hline & Unmarried & 5 & 01.9 \\
\hline & Separated & 5 & 01.9 \\
\hline \multirow{5}{*}{ Ethnicity } & Brahmin/Chhetri & 129 & 47.8 \\
\hline & Janajati & 95 & 35.2 \\
\hline & Muslim & 19 & 07.0 \\
\hline & Madheshi & 15 & 05.6 \\
\hline & Dalit & 12 & 04.4 \\
\hline \multirow{2}{*}{ Education status } & Literate & 189 & 70.0 \\
\hline & Illiterate & 81 & 30.0 \\
\hline \multirow{7}{*}{ Occupation } & Clerical, shop owner, farmer & 89 & 33.0 \\
\hline & Unemployed & 69 & 25.6 \\
\hline & Skilled worker & 42 & 15.6 \\
\hline & Semiprofession & 24 & 8.9 \\
\hline & Profession & 23 & 8.5 \\
\hline & Unskilled worker & 12 & 4.4 \\
\hline & Semiskilled worker & 11 & 4.1 \\
\hline \multirow{2}{*}{ Poverty line } & $<1.9$ USD & 61 & 22.6 \\
\hline & $>1.9$ USD & 209 & 77.4 \\
\hline \multirow{3}{*}{ Smoking tobacco products } & Current & 27 & 10.0 \\
\hline & Past & 31 & 11.5 \\
\hline & Never & 212 & 78.5 \\
\hline \multirow{4}{*}{ Alcohol consumption } & Never & 185 & 68.5 \\
\hline & Ex-alcoholic & 25 & 09.3 \\
\hline & Regular & 26 & 10.0 \\
\hline & Occasional & 34 & 12.6 \\
\hline \multirow{2}{*}{ Waist circumference } & Normal & 149 & 55.2 \\
\hline & High & 121 & 44.8 \\
\hline \multirow{4}{*}{ BMI } & Underweight & 4 & 1.5 \\
\hline & Normal & 59 & 21.9 \\
\hline & Overweight & 60 & 22.2 \\
\hline & Obese & 147 & 54.4 \\
\hline \multirow{2}{*}{$\mathrm{BP}$} & Yes & 86 & 32 \\
\hline & No & 184 & 68 \\
\hline \multirow{3}{*}{ Duration of diabetes } & $<5$ years & 142 & 52.6 \\
\hline & $5-10$ years & 91 & 33.7 \\
\hline & $>10$ years & 37 & 13.7 \\
\hline \multirow{2}{*}{ Other illness } & Yes & 155 & 57.4 \\
\hline & No & 115 & 42.6 \\
\hline \multirow{2}{*}{ Complication } & Yes & 51 & 18.9 \\
\hline & No & 219 & 81.1 \\
\hline \multirow{2}{*}{ Diabetes in family } & Yes & 167 & 61.9 \\
\hline & No & 103 & 38.1 \\
\hline \multirow{2}{*}{ Perception of disease } & Serious & 184 & 68.1 \\
\hline & Not serious & 86 & 31.9 \\
\hline \multirow{3}{*}{ On medication* } & OHA & 220 & 81.5 \\
\hline & Insulin & 19.0 & 6.7 \\
\hline & Both & 14.0 & 5.2 \\
\hline
\end{tabular}


Table 1: Continued.

\begin{tabular}{|c|c|c|c|}
\hline Characteristics & Categories & Frequency & Percentage (\%) \\
\hline \multirow{2}{*}{ Hospital visit } & Yes & 253 & 93.7 \\
\hline & No & 17 & 06.3 \\
\hline \multirow{2}{*}{ Private doctor visit } & Yes & 216 & 80.0 \\
\hline & No & 54 & 20.0 \\
\hline \multirow{2}{*}{ ER visit } & Yes & 22 & 08.1 \\
\hline & No & 248 & 91.9 \\
\hline \multirow{2}{*}{ Hospital admission } & Yes & 16 & 05.9 \\
\hline & No & 254 & 94.1 \\
\hline \multirow{2}{*}{ Awareness about PHC } & Yes & 154 & 57 \\
\hline & No & 116 & 43 \\
\hline \multirow{5}{*}{ Services available at $\mathrm{PHC} *$} & OPD & 152 & 99.3 \\
\hline & Immunization & 133 & 86.9 \\
\hline & Laboratory & 64 & 41.8 \\
\hline & Health education & 26 & 17.0 \\
\hline & Specific for diabetes & 19 & 12.4 \\
\hline \multirow{2}{*}{ Prefer PHC } & Yes & 5 & 3.3 \\
\hline & No & 148 & 96.7 \\
\hline
\end{tabular}

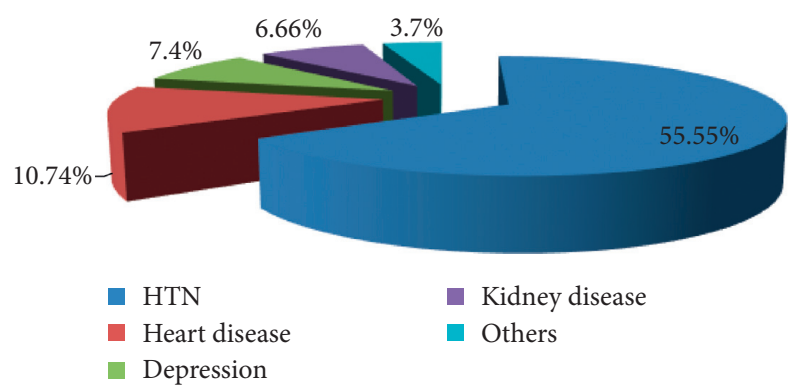

FIGURe 1: Prevalence of comorbidities among the participants; multiple response was provided by the participants with multiple diseases.

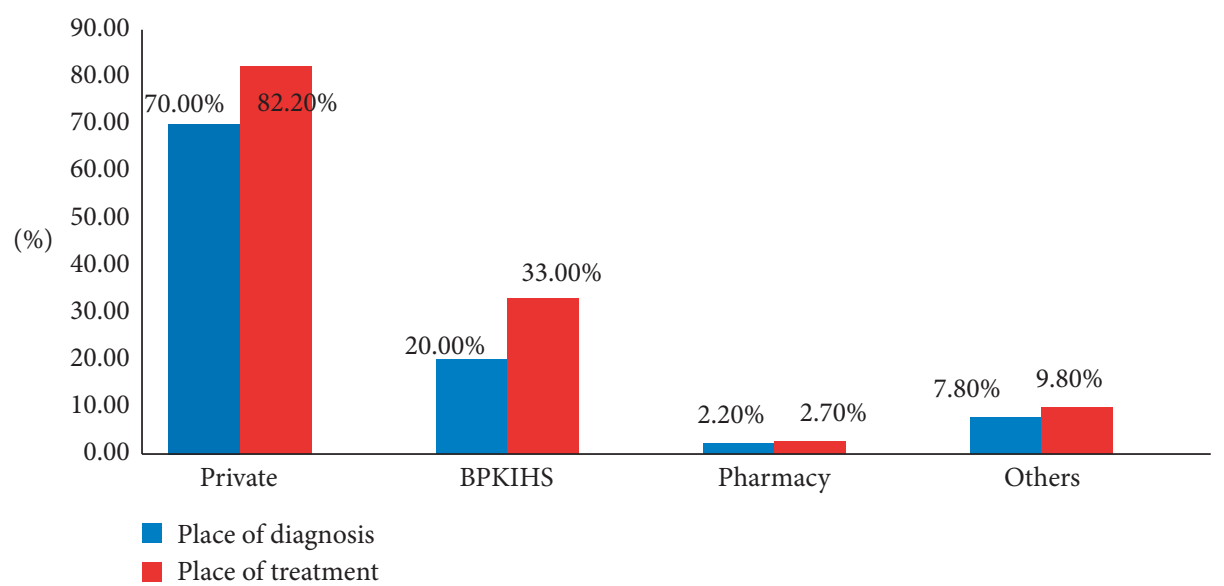

FIGURE 2: Distribution of participants according to place of diagnosis and treatment; multiple response was provided by the participants for visiting different health care facilities for diagnosis and treatment. 
TABle 2: Descriptive study of the D-39 questionnaire.

\begin{tabular}{lcccccc}
\hline D-39 domains & No. of items & Mean & Median & SD & Minimum & Maximum \\
\hline Energy and mobility & 15 & 38.40 & 38.09 & 9.29 & 11.90 & 62.38 \\
Diabetes control & 12 & 48.59 & 48.80 & 8.60 & 17.86 & 67.86 \\
Anxiety and worry & 4 & 57.34 & 57.14 & 11.08 & 14.29 & 12.86 \\
Social overload & 5 & 39.75 & 38.57 & 10.14 & 7.14 & 64.29 \\
Sexual behavior & 3 & 29.29 & 30.95 & 13.44 & 64.29 \\
Overall quality of life & 39 & 42.95 & 42.45 & 8.11 & 15.93 \\
\hline
\end{tabular}

TABLE 3: Association of demographic characteristics with QOL domains.

\begin{tabular}{|c|c|c|c|c|c|c|}
\hline Characteristics & $\begin{array}{c}\text { Energy and } \\
\text { mobility } \\
\text { mean } \pm \mathrm{SD}\end{array}$ & $\begin{array}{l}\text { Diabetes control } \\
\text { mean } \pm \mathrm{SD}\end{array}$ & $\begin{array}{c}\text { Anxiety and } \\
\text { worry mean } \pm \text { SD }\end{array}$ & $\begin{array}{l}\text { Social overload } \\
\text { mean } \pm \text { SD }\end{array}$ & $\begin{array}{c}\text { Sexual behavior } \\
\text { mean } \pm \text { SD }\end{array}$ & $\begin{array}{c}\text { Overall QOL } \\
\text { mean } \pm \text { SD }\end{array}$ \\
\hline \multicolumn{7}{|l|}{ Age in years } \\
\hline$<50$ & $33.40 \pm 8.028$ & $45.04 \pm 8.08$ & $54.04 \pm 10.57$ & $36.00 \pm 10.20$ & $26.91 \pm 12.89$ & $38.93 \pm 7.20$ \\
\hline$>50$ & $41.95 \pm 8.49$ & $51.10 \pm 8.08$ & $59.67 \pm 10.87$ & $42.42 \pm 9.24$ & $30.98 \pm 13.61$ & $45.80 \pm 7.50$ \\
\hline$p$ value & $<0.001$ & $<0.001$ & $<0.001$ & $<0.001$ & 0.014 & $<0.001$ \\
\hline \multicolumn{7}{|l|}{ Gender } \\
\hline Male & $38.13 \pm 9.79$ & $48.54 \pm 8.43$ & $56.32 \pm 11.37$ & $40.58 \pm 9.631$ & $29.90 \pm 13.83$ & $42.88 \pm 8.25$ \\
\hline Female & $38.68 \pm 8.79$ & $48.64 \pm 8.81$ & $58.36 \pm 10.72$ & $38.91 \pm 10.61$ & $28.67 \pm 13.06$ & $43.02 \pm 7.98$ \\
\hline$p$ value & 0.625 & 0.929 & 0.131 & 0.175 & 0.456 & 0.886 \\
\hline \multicolumn{7}{|l|}{ Ethnicity } \\
\hline Janajati & $36.45 \pm 8.83$ & $46.56 \pm 8.68$ & $55.52 \pm 11.89$ & $38.33 \pm 9.90$ & $29.19 \pm 13.45$ & $41.20 \pm 8.01$ \\
\hline Brahmin/Chhetri & $39.50 \pm 9.90$ & $49.52 \pm 8.67$ & $58.36 \pm 11.03$ & $39.81 \pm 10.35$ & $29.18 \pm 14.06$ & $43.77 \pm 8.43$ \\
\hline Others & $39.36 \pm 7.92$ & $50.15 \pm 7.63$ & $58.23 \pm 9.06$ & $42.54 \pm 9.66$ & $29.8 \pm 111.84$ & $44.29 \pm 6.81$ \\
\hline$p$ value & 0.038 & 0.015 & 0.140 & 0.068 & 0.960 & 0.030 \\
\hline \multicolumn{7}{|l|}{ Marital status } \\
\hline Unmarried & $30.76 \pm 10.09$ & $35.95 \pm 9.93$ & $47.14 \pm 8.14$ & $31.71 \pm 7.45$ & $19.52 \pm 9.87$ & $33.30 \pm 8.78$ \\
\hline Married & $38.54 \pm 9.24$ & $48.83 \pm 8.42$ & $57.53 \pm 11.05$ & $39.90 \pm 10.13$ & $29.47 \pm 13.45$ & $43.13 \pm 8.00$ \\
\hline$p$ value & 0.063 & 0.001 & 0.038 & 0.074 & 0.101 & 0.007 \\
\hline \multicolumn{7}{|l|}{ Education } \\
\hline Illiterate & $41.04 \pm 8.54$ & $49.83 \pm 8.12$ & $58.55 \pm 11.23$ & $42.27 \pm 9.83$ & $34.36 \pm 12.53$ & $45.18 \pm 7.77$ \\
\hline Literate & $37.27 \pm 9.40$ & $48.06 \pm 8.77$ & $56.82 \pm 11.01$ & $38.67 \pm 10.10$ & $27.12 \pm 13.26$ & $41.99 \pm 8.08$ \\
\hline$p$ value & 0.002 & 0.120 & 0.240 & 0.007 & $<0.001$ & 0.003 \\
\hline \multicolumn{7}{|l|}{ Occupation } \\
\hline Unemployed & $42.22 \pm 9.41$ & $50.65 \pm 9.78$ & $60.24 \pm 12.30$ & $42.87 \pm 9.68$ & $30.26 \pm 12.78$ & $45.83 \pm 8.80$ \\
\hline Employed & $37.09 \pm 8.90$ & $47.88 \pm 8.07$ & $56.34 \pm 10.48$ & $38.68 \pm 10.10$ & $28.96 \pm 13.68$ & $41.96 \pm 7.63$ \\
\hline$p$ value & $<0.001$ & 0.037 & 0.020 & 0.003 & 0.489 & 0.001 \\
\hline \multicolumn{7}{|l|}{ Poverty line } \\
\hline$<1.9$ & $37.93 \pm 09.28$ & $47.32 \pm 09.50$ & $57.14 \pm 12.52$ & $39.55 \pm 10.16$ & $28.84 \pm 13.31$ & $42.30 \pm 08.47$ \\
\hline$>1.9$ & $38.54 \pm 09.31$ & $48.96 \pm 08.31$ & $57.40 \pm 10.66$ & $39.81 \pm 10.16$ & $29.42 \pm 13.51$ & $43.14 \pm 08.01$ \\
\hline$p$ value & 0.651 & 0.192 & 0.874 & 0.860 & 0.767 & 0.476 \\
\hline \multicolumn{7}{|l|}{$\begin{array}{l}\text { Current smoking } \\
\text { status }\end{array}$} \\
\hline Yes & $37.23 \pm 8.86$ & $46.11 \pm 7.94$ & $53.17 \pm 11.48$ & $38.67 \pm 8.44$ & $27.60 \pm 14.21$ & $41.04 \pm 7.00$ \\
\hline No & $38.53 \pm 9.35$ & $48.86 \pm 8.65$ & $57.80 \pm 10.96$ & $38.87 \pm 10.32$ & $29.48 \pm 13.37$ & $43.17 \pm 8.20$ \\
\hline$p$ value & 0.490 & 0.116 & 0.039 & 0.499 & 0.491 & 0.198 \\
\hline \multicolumn{7}{|l|}{$\begin{array}{l}\text { Current alcohol } \\
\text { (drinking) status }\end{array}$} \\
\hline Yes & $35.09 \pm 8.10$ & $46.11 \pm 6.96$ & $53.63 \pm 10.51$ & $36.42 \pm 7.57$ & $26.11 \pm 12.62$ & $39.86 \pm 6.06$ \\
\hline No & $39.35 \pm 9.41$ & $49.30 \pm 8.91$ & $58.40 \pm 11.04$ & $40.70 \pm 10.60$ & $30.20 \pm 13.56$ & $43.83 \pm 8.41$ \\
\hline$p$ value & 0.002 & 0.004 & 0.003 & 0.001 & 0.037 & $<0.001$ \\
\hline \multicolumn{7}{|l|}{ Blood pressure } \\
\hline Normotensive & $37.22 \pm 9.38$ & $47.71 \pm 8.27$ & $56.30 \pm 10.67$ & $38.90 \pm 9.89$ & $28.22 \pm 13.24$ & $45.10 \pm 8.30$ \\
\hline Hypertensive & $40.89 \pm 8.64$ & $50.43 \pm 9.04$ & $59.52 \pm 11.66$ & $41.55 \pm 10.50$ & $31.55 \pm 13.65$ & $41.93 \pm 7.83$ \\
\hline$p$ value & 0.002 & 0.015 & 0.025 & 0.044 & 0.057 & 0.002 \\
\hline \multicolumn{7}{|l|}{$B M I\left(\mathrm{~kg} / \mathrm{m}^{2}\right)$} \\
\hline$<25$ & $39.64 \pm 9.67$ & $49.42 \pm 8.61$ & $59.03 \pm 11.66$ & $40.33 \pm 10.52$ & $29.67 \pm 13.90$ & $43.96 \pm 8.53$ \\
\hline
\end{tabular}


TABle 3: Continued.

\begin{tabular}{|c|c|c|c|c|c|c|}
\hline Characteristics & $\begin{array}{c}\text { Energy and } \\
\text { mobility } \\
\text { mean } \pm S D\end{array}$ & $\begin{array}{c}\text { Diabetes control } \\
\text { mean } \pm \mathrm{SD}\end{array}$ & $\begin{array}{c}\text { Anxiety and } \\
\text { worry mean } \pm \mathrm{SD}\end{array}$ & $\begin{array}{l}\text { Social overload } \\
\text { mean } \pm \text { SD }\end{array}$ & $\begin{array}{l}\text { Sexual behavior } \\
\text { mean } \pm S D\end{array}$ & $\begin{array}{c}\text { Overall QOL } \\
\text { mean } \pm \text { SD }\end{array}$ \\
\hline$>25$ & $37.37 \pm 8.87$ & $47.89 \pm 8.56$ & $55.92 \pm 10.41$ & $39.27 \pm 9.82$ & $28.97 \pm 13.09$ & $42.11 \pm 7.66$ \\
\hline$p$ value & 0.046 & 0.145 & 0.022 & 0.391 & 0.672 & 0.064 \\
\hline \multicolumn{7}{|c|}{$\begin{array}{l}\text { Duration of diabetes } \\
\text { (years) }\end{array}$} \\
\hline$<5$ & $34.88 \pm 8.10$ & $46.06 \pm 8.32$ & $55.48 \pm 9.78$ & $37.18 \pm 9.93$ & $26.45 \pm 12.91$ & $40.08 \pm 7.20$ \\
\hline$>5$ & $42.21 \pm 8.99$ & $51.39 \pm 8.05$ & $59.40 \pm 12.07$ & $42.61 \pm 9.63$ & $32.44 \pm 13.37$ & $46.13 \pm 7.90$ \\
\hline$p$ value & $<0.001$ & $<0.001$ & 0.004 & $<0.001$ & $<0.001$ & $<0.001$ \\
\hline \multicolumn{7}{|l|}{ Comorbidity } \\
\hline Yes & $41.65 \pm 8.54$ & $51.20 \pm 8.17$ & $59.49 \pm 11.17$ & $42.20 \pm 9.95$ & $32.05 \pm 13.92$ & $39.17 \pm 7.09$ \\
\hline No & $34.02 \pm 8.45$ & $45.07 \pm 7.92$ & $54.44 \pm 10.31$ & $36.45 \pm 9.48$ & $25.56 \pm 11.84$ & $45.75 \pm 7.68$ \\
\hline$p$ value & $<0.001$ & $<0.001$ & $<0.001$ & $<0.001$ & $<0.001$ & $<0.001$ \\
\hline \multicolumn{7}{|l|}{ Complication } \\
\hline Yes & $45.44 \pm 8.96$ & $53.96 \pm 7.64$ & $63.16 \pm 11.37$ & $44.95 \pm 8.70$ & $31.60 \pm 13.33$ & $48.75 \pm 7.58$ \\
\hline No & $36.77 \pm 8.60$ & $47.34 \pm 8.35$ & $55.98 \pm 10.60$ & $38.54 \pm 10.09$ & $28.75 \pm 13.44$ & $41.60 \pm 7.63$ \\
\hline$p$ value & $<0.001$ & $<0.001$ & $<0.001$ & $<0.001$ & 0.173 & $<0.001$ \\
\hline \multicolumn{7}{|c|}{$\begin{array}{l}\text { Family history of } \\
\text { diabetes }\end{array}$} \\
\hline Yes & $39.09 \pm 9.82$ & $50.11 \pm 8.48$ & $59.30 \pm 10.82$ & $40.33 \pm 10.06$ & $28.78 \pm 13.92$ & $43.92 \pm 8.34$ \\
\hline No & $37.29 \pm 8.29$ & $46.12 \pm 8.26$ & $54.16 \pm 10.80$ & $38.82 \pm 10.24$ & $30.12 \pm 12.65$ & $41.38 \pm 7.50$ \\
\hline$p$ value & 0.109 & $<0.001$ & $<0.001$ & 0.235 & 0.429 & 0.012 \\
\hline $\mathrm{OHA}$ & $38.25 \pm 8.68$ & $48.82 \pm 7.60$ & $57.37 \pm 10.13$ & $39.68 \pm 9.79$ & $29.57 \pm 13.28$ & $42.11 \pm 6.33$ \\
\hline Insulin & $45.63 \pm 8.40$ & $54.41 \pm 6.989$ & $63.86 \pm 11.07$ & $44.45 \pm 8.65$ & $26.19 \pm 14.28$ & $48.51 \pm 6.50$ \\
\hline Both & $44.90 \pm 8.79$ & $55.52 \pm 5.63$ & $65.05 \pm 9.62$ & $47.14 \pm 9.50$ & $37.75 \pm 10.00$ & $47.44 \pm 4.99$ \\
\hline$p$ value & $<0.001$ & $<0.001$ & 0.002 & 0.005 & 0.442 & 0.025 \\
\hline \multicolumn{7}{|c|}{$\begin{array}{l}\text { Hospital visit in last } 6 \\
\text { months }\end{array}$} \\
\hline Yes & $38.72 \pm 8.98$ & $49.11 \pm 7.85$ & $57.72 \pm 10.38$ & $39.77 \pm 10.05$ & $29.39 \pm 13.25$ & $43.28 \pm 7.60$ \\
\hline No & $33.70 \pm 12.51$ & $40.82 \pm 14.33$ & $51.68 \pm 18.21$ & $39.41 \pm 11.77$ & $27.8 \pm 17.65$ & $38.01 \pm 12.90$ \\
\hline$p$ value & 0.031 & 0.031 & 0.194 & 0.885 & 0.732 & 0.115 \\
\hline \multicolumn{7}{|c|}{$\begin{array}{l}\text { Private doctor in last } \\
6 \text { months }\end{array}$} \\
\hline Yes & $38.46 \pm 8.93$ & $48.97 \pm 7.94$ & $57.45 \pm 10.12$ & $39.97 \pm 9.92$ & $28.83 \pm 12.70$ & $43.09 \pm 7.52$ \\
\hline No & $38.18 \pm 10.72$ & $47.06 \pm 10.81$ & $56.88 \pm 14.40$ & $38.89 \pm 11.02$ & $31.12 \pm 16.10$ & $42.38 \pm 10.20$ \\
\hline$p$ value & 0.845 & 0.228 & 0.781 & 0.483 & 0.334 & 0.630 \\
\hline \multicolumn{7}{|c|}{$\begin{array}{l}\text { ER visit in last } 6 \\
\text { months }\end{array}$} \\
\hline Yes & $44.80 \pm 8.51$ & $54.76 \pm 8.24$ & $63.31 \pm 11.36$ & $45.84 \pm 9.64$ & $33.33 \pm 13.60$ & $49.01 \pm 7.84$ \\
\hline No & $37.84 \pm 9.17$ & $48.04 \pm 8.44$ & $56.81 \pm 10.92$ & $39.21 \pm 10.02$ & $28.93 \pm 13.40$ & $42.41 \pm 7.92$ \\
\hline$p$ value & 0.001 & $<0.001$ & 0.008 & 0.003 & 0.142 & $<0.001$ \\
\hline \multicolumn{7}{|c|}{$\begin{array}{l}\text { Hospital admission in } \\
\text { last } 6 \text { months }\end{array}$} \\
\hline Yes & $43.87 \pm 11.43$ & $52.68 \pm 11.96$ & $60.50 \pm 15.90$ & $44.82 \pm 11.53$ & $34.52 \pm 13.85$ & $47.68 \pm 11.20$ \\
\hline No & $38.06 \pm 9.06$ & $48.33 \pm 8.31$ & $57.14 \pm 10.72$ & $39.43 \pm 9.99$ & $28.96 \pm 13.38$ & $42.65 \pm 7.80$ \\
\hline$p$ value & 0.015 & 0.050 & 0.242 & 0.039 & 0.109 & 0.016 \\
\hline
\end{tabular}

that although the disease is socially being acceptable, the patients are worried about their future health outcomes and economic burden.

After adjusting for the other variables, having age more than 50 years was associated with affected quality of life in the domain: energy and mobility $(<0.001)$, diabetes control $(<0.001)$, anxiety and worry $(<0.001)$ and social overload $(<0.001)$, and overall QOL by $5.460,4.249,4.237,4.030$, and 3.970 , respectively. Similarly, an earlier study done in Riyadh, Saudi Arabia, found the group with over 50 years of age was significantly associated with lower quality of life on subscale physical functioning $(p<0.001)$ and role of emotional $(p<0.01)$ [14]. The study in US adults showed quality of life, in which domain physical functioning and social functioning were affected with increase in age, but in mental health, it is affected in the younger group [15]. The similar finding was found in the study done among Dutch patients, where older patients reported lower quality of life than the younger one [16]. This signifies as the people become old, physical activity is low, associated with different comorbidities and complications and worried about their health-related outcomes. 
TABLE 4: Multiple linear regression analysis of significant factors associated with QOL and its domains.

\begin{tabular}{|c|c|c|c|}
\hline QOL and its domain variables & Unstandardized coefficients & $95 \% \mathrm{CI}$ & $p$ value \\
\hline \multicolumn{4}{|l|}{ Energy and mobility } \\
\hline Age & 5.460 & $3.504-7.415$ & $<0.001$ \\
\hline Education & 2.421 & $0.50-4.34$ & 0.014 \\
\hline Current alcoholic & -2.78 & $-4.90-(-0.65)$ & 0.011 \\
\hline Duration of diabetes in years & 2.69 & $2.74-4.63$ & 0.007 \\
\hline Comorbidity & 2.58 & $0.57-4.60$ & 0.012 \\
\hline Complication & 5.937 & $3.62-8.25$ & $<0.001$ \\
\hline Hospital visit & 4.70 & $1.143-8.25$ & 0.010 \\
\hline ER visit & 4.60 & $1.43-7.76$ & 0.005 \\
\hline Constant & 26.447 & & \\
\hline \multicolumn{4}{|l|}{ Diabetes control } \\
\hline Age & 4.25 & $2.37-6.12$ & $<0.001$ \\
\hline Marital status & 8.87 & $2.44-15.30$ & 0.007 \\
\hline Current alcoholic & -2.60 & $-4.70-(-0.52)$ & 0.014 \\
\hline Comorbidity & 2.33 & $0.37-4.30$ & 0.020 \\
\hline Complication & 4.48 & $2.17-6.80$ & $<0.001$ \\
\hline Family history & 1.60 & $-0.24-3.45$ & 0.088 \\
\hline Hospital visit & 6.96 & $3.36-10.55$ & $<0.001$ \\
\hline ER visit & 4.36 & $1.20-7.52$ & 0.007 \\
\hline Constant & 27.919 & & \\
\hline \multicolumn{4}{|l|}{ Anxiety and worry } \\
\hline Age & 4.24 & $1.76-6.71$ & 0.001 \\
\hline Marital status & 8.70 & $-0.37-(-17.74)$ & 0.060 \\
\hline Current alcoholic & -3.65 & $-6.61-(-0.70)$ & 0.016 \\
\hline BMI & -2.65 & $-5.12-(0.17)$ & 0.036 \\
\hline Complication & 5.18 & $2.01-8.35$ & 0.001 \\
\hline Family history & 3.66 & $1.11-6.21$ & 0.005 \\
\hline ER visit & 5.03 & $0.58-9.47$ & 0.027 \\
\hline Constant & 44.93 & & \\
\hline \multicolumn{4}{|l|}{ Social overload } \\
\hline Age & 4.03 & $1.61-6.45$ & 0.001 \\
\hline Education & 2.86 & $0.43-5.30$ & 0.021 \\
\hline Current alcoholic & -3.63 & $-5.33-(0.89)$ & 0.006 \\
\hline Comorbidity & 2.79 & $0.311-5.27$ & 0.028 \\
\hline Complication & 4.41 & $1.53-7.30$ & 0.003 \\
\hline ER visit & 5.64 & $1.66-9.63$ & 0.006 \\
\hline Constant & 35.84 & & \\
\hline \multicolumn{4}{|l|}{ Sexual behavior } \\
\hline Education & 7.46 & $4.15-10.76$ & $<0.001$ \\
\hline Disease duration & 2.86 & $-0.41-6.12$ & 0.086 \\
\hline Comorbidity & 5.65 & $2.37-8.92$ & 0.001 \\
\hline Constant & 24.276 & & \\
\hline \multicolumn{4}{|l|}{ Overall QOL } \\
\hline Age & 3.97 & $2.21-5.72$ & $<0.001$ \\
\hline Marital status & 7.02 & $1.22-12.82$ & 0.018 \\
\hline Education & 1.90 & $0.17-3.68$ & 0.032 \\
\hline Current alcoholic & -2.115 & $-4.05-(-0.18)$ & 0.032 \\
\hline BMI & -1.40 & $-3.03-0.23$ & $0 . .092$ \\
\hline Disease duration & 1.84 & $0.09-3.60$ & 0.039 \\
\hline Comorbidity & 2.81 & $0.97-4.64$ & 0.003 \\
\hline Complication & 4.76 & $2.70-6.83$ & $<0.001$ \\
\hline ER visit & 4.94 & $2.12-7.78$ & 0.001 \\
\hline Constant & 30.615 & & \\
\hline
\end{tabular}

Significant association was found with increase in the duration of disease in the domain: energy and mobility ( $p=$ $0.007)$ and overall QOL (0.039). The quality of life was affected by 2.686 and 1.845 , respectively, in those who were suffering from the disease for more than 5 years. This was comparable with the study done in Saudi Arabia patients concluding that the longer duration of disease is associated with poor quality of life [14]. This was also consistent with 
the finding from the study done among females with type 2 diabetes referred to the Diabetes Clinic of Khoy City, Northwest of Iran [17]. This infers that the incidence of diabetes complications rises with the increase in the disease duration, which in turn, negatively affects the patients' QOL. However, also a contrary result was found in the study done in Kathmandu, Nepal, explaining having diagnosed for more than 10 years increased the quality of life in physical health domain by 5.184 score [13]. An observational study carried out in Dr. Sardjito Hospital in Yogyakarta, Indonesia, demonstrated no significant association between disease duration and the diabetes QOL clinical trial questionnaire [18].

The presence of comorbidity was found to be significantly associated with quality of life affected in the domain of energy and mobility $(p=0.012)$, diabetes control $(p=0.020)$, social overload $(p=0.028)$, sexual behavior $(p=0.001)$, and overall QOL $(0.003)$ by $2.583,2.334,2.789$, 5.649 , and 2.807 , respectively. This finding was similar with the finding of the study done in US adults, which showed that with the presence and the number of comorbidity, quality of life is affected in the domain of physical functioning, social functioning, and mental health [15]. The study done among females in Iran also found the consistent finding [17]. However, also a contrary result was found for the mental health domain which was not significant in the present study.

Those participants having an emergency room visit in last 6 months showed that the quality of life was affected in the domain: energy and mobility $(p=0.005)$, diabetes control $(p=0.007)$, anxiety and worry $(p=0.027)$, social overload $(p=0.006)$, and overall QOL $(p=0.001)$ by 4.596 , $4.364,5.026,5.644$, and 4.948, respectively. The study done among US adults with diabetes indicated that limited access to healthcare especially low frequency or no use of healthcare services was associated with poor glycemic control, which may lead to poor quality of life [19]. This finding was contrary with the finding of the study done among US Shield surveys, where no association was found between ER visit and quality of life of the diabetes patients [5].

Those participants having history of hospital admission in last 6 months showed poor QOL in the domain, of energy and mobility, social overload, and overall QOL. This was comparable with the finding of a study done among US adults having diabetes and was a part of the marketing company using the SF-20 questionnaire (physical function, social function, and mental health) [15].

\section{Conclusions}

In this study, the quality of life of the participants was more affected in the domain of anxiety and worry with the highest mean score (SD) $(57.34 \pm 11.08)$. The utilization of private health facilities is high for both diagnosis (70\%) and treatment $(82.2 \%)$, which concludes that people in this region are more aware about their health outcomes.

Age, marital status, education, alcohol consumption status, duration of disease, comorbidity, presence of complications, and emergency room visits were significantly associated with overall QOL.

\section{Data Availability}

The data used to support the findings of this study are available from the corresponding author upon request.

\section{Conflicts of Interest}

The authors declare no conflicts of interest.

\section{Acknowledgments}

The authors thank Female Community Health Volunteers and Community Health System Innovation (COHESION)Project for help in search of people living with type 2 diabetes during door-to-door visits.

\section{References}

[1] WHO, Multisectoral Action Plan for the Prevention and Control of Non Communicable Diseases (2014-2020) Government of Nepal, WHO, Kathmandu, Nepal, 2014.

[2] B. Gyawali, A. Ferrario, and E. Teijlingen, "Challenges in diabetes mellitus type 2 management in Nepal: a literature review," Global Health Action, vol. 1, pp. 1-12, 2016.

[3] S. R. Upreti, G. R. Lohani, and L. P. Dixit, "Strengthening policy and governance to address the growing burden of diabetes in Nepal," WHO South-East Asia Journal of Public Health, vol. 5, no. 1, pp. 40-43, 2016.

[4] S. Mohammadi, N. Karim, R. Abd.Talib, and R. Amani, "Evaluation of quality of life among type 2 diabetes patients," International Journal of Community Medicine and Public Health, vol. 3, no. 1, pp. 51-56, 2016.

[5] A. J. Green, D. D. Bazata, K. M. Fox, and S. Grandy, "Quality of life, depression, and healthcare resource utilization among adults with type 2 diabetes mellitus and concomitant hypertension and obesity: a prospective survey," Cardiology Research and Practice, vol. 2012, Article ID 404107, 15 pages, 2012.

[6] J. Ha, J. Takala, and S. Keina, "Good continuity of care may improve quality of life in type 2 diabetes," Diabetes Research and Clinical Practice, vol. 51, no. 1, pp. 21-27, 2001.

[7] R. Mehta, S. Subedi, and S. Bohora, "Health related quality of life of diabetic patients visited in Koshi zonal hospital, Biratnagar," Journal of Chitwan Medical College, vol. 4, no. 9, pp. 13-16, 2014.

[8] A. Osman Mahgoub and E. Abdelgadir, "The association between health-related quality of life and ramadan fasting in diabetic patients: a survey using a structured D-39 assessment tool. a sudanese cohort," Journal of Fasting Health, vol. 5, no. 1, pp. 24-30, 2017, http://jfh.mums.ac.ir/article_8343_ a7d4add2430f65ed53df6563c76ae103.pdf.

[9] M. Riaz, R. a. Rehman, R. Hakeem, and F. Shaheen, "Health related quality of life in patients with diabetes using SF-12 questionnaire," Journal of Diabetology, vol. 2, pp. 1-7, 2013.

[10] F. Nouh, M. Omar, and M. Younis, "Prevalence of hypertension among diabetic patients in Benghazi: a study of associated factors," Asian Journal of Medicine and Health, vol. 6, no. 4, pp. 1-11, 2017.

[11] S. Deshmukh, S. K. Kalaskar, S. B. Kadam et al., "Utilization pattern of health services for non-communicable diseases in an urban slum: a study of Turbhe stores slum in Navi Mumbai, 
Maharashtra, India," International Journal of Community Medicine And Public Health, vol. 4, no. 1, p. 139, 2016, http:// www.ijcmph.com/index.php/ijcmph/article/view/26.

[12] R. Z. Luana, d. S. Manoel Antônio, V. Vívian Saraiva, L. R. Flávia Fernanda, A. A. Clarissa Cordeiro, and Z. Maria Lucia, "Quality of life in patients with diabetes using the diabetes 39 (d-39) instrument," Revista Gaúcha de Enfermagem, vol. 39, no. 3, pp. 138-146, 2013.

[13] S. R. Mishra, A. Sharma, P. M. Bhandari, S. Bhochhibhoya, and K. Thapa, "Depression and health-related quality of life among patients with type 2 diabetes mellitus: a cross-sectional study in Nepal," PLoS One, vol. 10, no. 11, pp. 1-13, 2015.

[14] A. A. Al Hayek, A. A. Robert, A. Al Saeed, A. A. Alzaid, and F. S. Al Sabaan, "Factors associated with health-related quality of life among Saudi patients with type 2 diabetes mellitus: a cross-sectional survey," Diabetes \& Metabolism Journal, vol. 38, no. 3, pp. 220-229, 2014, http://www. pubmedcentral.nih.gov/articlerender.fcgi? artid $=4083029 \&$ tool $=$ pmcentrez\&rendertype $=$ abstract .

[15] R. Island, N. Brunswick, D. Control, and C. Trial, "Quality of life and associated sample of adults with diabetes," Diabetes, vol. 20, no. 4, pp. 562-567, 1997.

[16] W. K. Redekop, M. a. Koopmanschap, R. P. Stolk, G. E. H. M. Rutten, B. H. R. Wolffenbuttel, and L. W. Niessen, "Health-related quality of life and treatment satisfaction in Dutch patients with type 2 diabetes," Diabetes Care, vol. 25, pp. 458-463, 2002.

[17] A. Didarloo and M. Alizadeh, "Health-related quality of life and its determinants amongst women with diabetes mellitus: a crosssectional analysis," Nursing and Midwifery Studies, vol. 5, no. 1, http://www.nmsjournal.com/?page=article\&article_id=28937, Article ID e2893, 2016.

[18] T. M. Andayani, M. I. M. Ibrahim, and A. H. Asdie, "The association of diabetes-related factor and quality of life in type 2 diabetes mellitus," International Journal of Pharmacy and Pharmaceutical Sciences, vol. 2, no. 1, pp. 139-145, 2010.

[19] X. Zhang, K. M. Bullard, E. W. Gregg et al., "Access to health care and control of ABCS of diabetes," Diabetes Care, vol. 35, no. 7, pp. 1566-1571, 2012. 